\title{
NUMERICAL SOLUTIONS OF STOCHASTIC FUNCTIONAL DIFFERENTIAL EQUATIONS
}

\author{
XUERONG MAO
}

\begin{abstract}
In this paper, the strong mean square convergence theory is established for the numerical solutions of stochastic functional differential equations (SFDEs) under the local Lipschitz condition and the linear growth condition. These two conditions are generally imposed to guarantee the existence and uniqueness of the true solution, so the numerical results given here were obtained under quite general conditions.
\end{abstract}

\section{Introduction}

Stochastic differential equations (SDEs) arise in mathematical models of physical systems that possess inherent noise and uncertainty. Such models have been used with great success in a variety of application areas, including biology, epidemiology, mechanics, economics and finance. Most SDEs arising in practice are nonlinear, and cannot be solved explicitly, so the construction of efficient computational methods is of great importance. A substantial body of work has been done concerning approximate schemes for SDEs. Most of the existing convergence theory for numerical methods requires a global Lipschitz assumption on the drift and diffusion coefficients of SDEs; see $[10,15,17,18]$, for example. Recent work has studied convergence in probability [3, 5] and almost sure convergence [4], under more relaxed conditions on the coefficients. In particular, [7] has discussed the strong mean square convergence for the Euler-Maruyama method, requiring only that the SDE be locally Lipschitz and that the $p$ th moments of the exact and numerical solution be bounded for some $p>2$. Moreover, [8] also considered the backward Euler method for SDEs without global Lipschitz assumptions.

In many applications where SDEs are used, one assumes that the system under consideration is governed by a principle of causality; that is, the future state of the system is independent of the past states, and is determined solely by the present. Under closer scrutiny, however, it becomes apparent that the principle of causality is often only a first approximation to the true situation, and that a more realistic model would include some of the past states of the system. Stochastic functional differential equations (SFDEs) give a mathematical formulation for such systems (see, for example, [6]). A special and important class of SFDEs is the stochastic differential delay equations (SDDEs)

$$
d x(t)=F(x(t), x(t-\tau)) d t+G(x(t), x(t-\tau)) d B(t) .
$$

As SDEs, most SDDEs cannot be solved explicitly, so numerical methods for SDDEs have recently received a great deal of attention (see, for example, $[1,2,9,12,13,14]$ ). Most of the existing convergence theory for numerical methods requires $F$ and $G$ to be globally 
Lipschitz. However, there are many SDDEs that do not satisfy the global Lipschitz condition; for example,

$$
d x(t)=x(t)[1+\sin (x(t-\tau))] d t+x(t)[1+\cos (x(t-\tau))] d B(t)
$$

satisfies only the local Lipschitz condition. It is in this spirit that recent work [16] has studied the Euler-Maruyama scheme for SDDEs under the local Lipschitz condition.

On the other hand, little is as yet known about numerical solutions for SFDEs. In this paper we study the Euler-Maruyama numerical solutions of the SFDE

$$
d x(t)=f\left(x_{t}\right) d t+g\left(x_{t}\right) d B(t), \quad t \geqslant 0,
$$

with initial data $x_{0}=\xi \in L_{\mathcal{F}_{0}}^{p}\left([-\tau, 0] ; R^{n}\right)$. Here,

$$
f: C\left([-\tau, 0] ; R^{n}\right) \rightarrow R^{n}, \quad g: C\left([-\tau, 0] ; R^{n}\right) \rightarrow R^{n \times m},
$$

$x(t) \in R^{n}$ for each $t$,

$$
x_{t}=\{x(t+\theta):-\tau \leqslant \theta \leqslant 0\} \in C\left([-\tau, 0] ; R^{n}\right),
$$

and $B(t)$ is an $m$-dimensional Brownian motion. The initial data $\xi$ is an $\mathcal{F}_{0}$-measurable $C\left([-\tau, 0] ; R^{n}\right)$-valued random variable such that $E\|\xi\|^{p}<\infty$ for some $p>2$. Our primary objective is to study strong mean-square convergence questions for the EulerMaruyama numerical approximations in the case where $f$ and $g$ satisfy both the local Lipschitz condition and the linear growth condition. These two conditions are in general imposed to guarantee the existence and uniqueness of the true solution, so our numerical results are obtained under rather general conditions.

It is perhaps useful to emphasise the local Lipschitz condition. Technically speaking, most of the existing convergence theory for the Euler-Maruyama numerical method requires the global Lipschitz condition, even when the underlying equations are SDEs (see, for example, $[10,15,17,18]$ ) or SDDEs (see, for example, $[1,2,9,12,13,14])$. Only recently have $[7,8]$ and [16] studied the strong mean-square convergence for the Euler-Maruyama numerical solutions for SDEs and SDDEs, respectively, without global Lipschitz assumptions. In other words, the new numerical techniques for SDEs and SDDEs under the local Lipschitz condition have only recently been developed. In this paper we generalize these techniques to deal with much more general SFDEs.

In Section 2, we introduce the Euler-Maruyama method for SFDEs, and we state our main result: that the Euler-Maruyama numerical solutions converge strongly to the exact solution if $f$ and $g$ satisfy the local Lipschitz condition and the linear growth condition. The proof of this result is rather technical, so we prepare a number of lemmas in Section 3, and then complete the proof in Section 4. In Section 5, we reveal the order of the convergence of the numerical solutions to the true solutions if the local Lipschitz condition is strengthened to the global Lipschitz condition. We show that the order is halved, which is the same as in the case of SDEs. In Section 6, we point out that our main result remains true if the linear growth condition is replaced by the condition that the exact and numerical solution have bounded $p$ th moment for some $p>2$. The bounded moment assumption will not, of course, hold in general, although it holds under the linear growth condition. It is an open problem to find other conditions, instead of the linear growth condition, that guarantee the bounded moment for the numerical solution. In Section 7, a simpler numerical scheme is presented in the case where both coefficients of the underlying SFDE are defined for step functions as well. 


\section{The Euler-Maruyama method}

Throughout this paper, unless otherwise specified, we use the following notations. Let $|\cdot|$ be the Euclidean norm in $R^{n}$. If $A$ is a vector or matrix, its transpose is denoted by $A^{T}$. If $A$ is a matrix, its trace norm is denoted by

$$
|A|=\sqrt{\operatorname{trace}\left(A^{T} A\right)}
$$

Let $R_{+}=[0, \infty)$, and let $\tau>0$. Denote by $C\left([-\tau, 0] ; R^{n}\right)$ the family of continuous functions from $[-\tau, 0]$ to $R^{n}$ with the norm $\|\varphi\|=\sup _{-\tau \leqslant \theta \leqslant 0}|\varphi(\theta)|$.

Let $\left(\Omega, \mathcal{F},\left\{\mathcal{F}_{t}\right\}_{t} \geqslant 0, P\right)$ be a complete probability space with a filtration $\left\{\mathcal{F}_{t}\right\}_{t} \geqslant 0$ satisfying the usual conditions (that is, it is increasing and right continuous, while $\mathcal{F}_{0}$ contains all $P$-null sets). Let $B(t)=\left(B_{1}(t), \ldots, B_{m}(t)\right)^{T}$ be an $m$-dimensional Brownian motion defined on the probability space. Let $p>0$, and denote by $L_{\mathcal{F}_{0}}^{p}\left([-\tau, 0] ; R^{n}\right)$ the family of $\mathcal{F}_{0}$-measurable $C\left([-\tau, 0] ; R^{n}\right)$-valued random variables such that $E\|\xi\|^{p}<\infty$. If $x(t)$ is an $R^{n}$-valued stochastic process on $t \in[-\tau, \infty)$, we let $x_{t}=\{x(t+\theta):-\tau \leqslant \theta \leqslant 0\}$ for $t \geqslant 0$.

Let $f: C\left([-\tau, 0] ; R^{n}\right) \rightarrow R^{n}$ and $g: C\left([-\tau, 0] ; R^{n}\right) \rightarrow R^{n \times m}$. In this paper we impose the following hypotheses.

AsSUMPTION 2.1 (THE LOCAL LiPSCHITZ CONDITION). For each integer $j \geqslant 1$, there is a right-continuous nondecreasing function $\mu_{j}:[-\tau, 0] \rightarrow R_{+}$such that

$$
|f(\varphi)-f(\psi)|^{2} \vee|g(\varphi)-g(\psi)|^{2} \leqslant \int_{-\tau}^{0}|\varphi(\theta)-\psi(\theta)|^{2} d \mu_{j}(\theta)
$$

for those $\varphi, \psi \in C\left([-\tau, 0] ; R^{n}\right)$ with $\|\varphi\| \vee\|\psi\| \leqslant j$, where the integral is of the LebesgueStieltjes type.

AsSUMPtion 2.2 (THE LINEAR GROWTH CONDITION). There is a constant $K>0$ such that

$$
|f(\varphi)|^{2} \vee|g(\varphi)|^{2} \leqslant K\left(1+\|\varphi\|^{2}\right)
$$

for all $\varphi \in C\left([-\tau, 0] ; R^{n}\right)$.

Consider the $n$-dimensional SFDE:

$$
d x(t)=f\left(x_{t}\right) d t+g\left(x_{t}\right) d B(t), \quad t \geqslant 0,
$$

with initial data $x_{0}=\xi$. We impose the following condition on the initial data.

Assumption 2.3. $\xi \in L_{\mathcal{F}_{0}}^{p}\left([-\tau, 0] ; R^{n}\right)$ for some $p>2$.

By [15, Theorem 5.2.5 on p. 153] and [15, Theorem 5.4.1 on p. 158], we can therefore state the following theorem.

THEOREM 2.4. Under Assumptions 2.1-2.3, equation (2.1) has a unique continuous solution $x(t)$ on $t \geqslant-\tau$. Moreover, the solution has the property that

$$
E\left(\sup _{-\tau \leqslant t \leqslant T}|x(t)|^{p}\right) \leqslant 2^{(p+4) / 2}\left(1+E\|\xi\|^{p}\right) e^{C T}
$$

for any $T>0$, where $C=p[2 \sqrt{K}+(33 p-1) K]$. In other words, the pth moment of the solution is finite. 
Let us now introduce a numerical scheme for the SFDE (2.1); we refer to it as the EulerMaruyama method. Let the step size $\Delta \in(0,1)$ be a fraction of $\tau$, namely $\Delta=\tau / N$ for some integer $N>\tau$. The discrete Euler-Maruyama approximate solution $\bar{y}(k \Delta), k \geqslant-N$ is defined as follows:

$$
\begin{cases}\bar{y}(k \Delta)=\xi(k \Delta), & -N \leqslant k \leqslant 0, \\ \bar{y}((k+1) \Delta)=\bar{y}(k \Delta)+f\left(\bar{y}_{k \Delta}\right) \Delta+g\left(\bar{y}_{k \Delta}\right) \Delta B_{k}, \quad k \geqslant 0,\end{cases}
$$

where $\Delta B_{k}=B((k+1) \Delta)-B(k \Delta)$ and $\bar{y}_{k \Delta}=\left\{\bar{y}_{k \Delta}(\theta):-\tau \leqslant \theta \leqslant 0\right\}$ is a $C\left([-\tau, 0] ; R^{n}\right)$-valued random variable defined as follows:

$$
\begin{aligned}
\bar{y}_{k \Delta}(\theta)=\bar{y}((k+i) \Delta)+ & \frac{\theta-i \Delta}{\Delta}[\bar{y}((k+i+1) \Delta)-\bar{y}((k+i) \Delta)] \\
& \text { for } i \Delta \leqslant \theta \leqslant(i+1) \Delta, i=-N,-(N-1), \ldots,-1 .
\end{aligned}
$$

That is, $\bar{y}_{k \Delta}(\cdot)$ is the linear interpolation of $\bar{y}((k-N) \Delta), \bar{y}((k-N+1) \Delta), \ldots, \bar{y}(k \Delta)$. We can re-write (2.4) as

$$
\bar{y}_{k \Delta}(\theta)=\frac{\Delta-(\theta-i \Delta)}{\Delta} \bar{y}((k+i) \Delta)+\frac{\theta-i \Delta}{\Delta} \bar{y}((k+i+1) \Delta),
$$

which yields

$$
\begin{aligned}
\left|\bar{y}_{k \Delta}(\theta)\right| & =\frac{\Delta-(\theta-i \Delta)}{\Delta}|\bar{y}((k+i) \Delta)|+\frac{\theta-i \Delta}{\Delta}|\bar{y}((k+i+1) \Delta)| \\
& \leqslant|\bar{y}((k+i) \Delta)| \vee|\bar{y}((k+i+1) \Delta)| .
\end{aligned}
$$

We therefore have

$$
\left\|\bar{y}_{k \Delta}\right\|=\max _{-N \leqslant i \leqslant 0}|\bar{y}(k+i) \Delta|, \quad \forall k \geqslant 0 .
$$

In our analysis it will be more convenient to use continuous-time approximations. We hence introduce the $C\left([-\tau, 0] ; R^{n}\right)$-value step process

$$
\bar{y}_{t}=\sum_{k=0}^{\infty} \bar{y}_{k \Delta} 1_{[k \Delta,(k+1) \Delta)}(t), \quad t \geqslant 0,
$$

and we define the continuous Euler-Maruyama approximate solution as follows:

$$
y(t)= \begin{cases}\xi(t), & -\tau \leqslant t \leqslant 0, \\ \xi(0)+\int_{0}^{t} f\left(\bar{y}_{s}\right) d s+\int_{0}^{t} g\left(\bar{y}_{s}\right) d B(s), \quad t \geqslant 0 .\end{cases}
$$

It should be pointed out that the $C\left([-\tau, 0] ; R^{n}\right)$-value process $\bar{y}_{t}$ is simply defined by (2.6), but we do not define here an $R^{n}$-valued continuous process $\bar{y}(t)$ from which $\bar{y}_{t}$ is then induced by $\bar{y}_{t}=\{\bar{y}(t+\theta):-\tau \leqslant \theta \leqslant 0\}$. It should also be pointed out that the reason why we do not use the linear interpolation of $\bar{y}(k \Delta)$ as a continuous-time approximation for $x(t)$, instead using $y(t)$ from $(2.7)$, is because the linear interpolation of $\bar{y}(k \Delta)$ is not $\mathcal{F}_{t}$-adapted.

It follows from (2.7) that for any $t \geqslant 0$ and any integer $k \geqslant 0$ that satisfy $k \Delta \leqslant t$,

$$
\begin{aligned}
y(t) & =\xi(0)+\int_{0}^{k \Delta} f\left(\bar{y}_{s}\right) d s+\int_{0}^{k \Delta} g\left(\bar{y}_{s}\right) d B(s)+\int_{k \Delta}^{t} f\left(\bar{y}_{s}\right) d s+\int_{k \Delta}^{t} g\left(\bar{y}_{s}\right) d B(s) \\
& =\bar{y}(k \Delta)+\int_{k \Delta}^{t} f\left(\bar{y}_{s}\right) d s+\int_{k \Delta}^{t} g\left(\bar{y}_{s}\right) d B(s) .
\end{aligned}
$$


In particular, we observe that $y(k \Delta)=\bar{y}(k \Delta)$ for all $k \geqslant-N$. That is, the discrete and continuous Euler-Maruyama approximate solutions coincide at the gridpoints. It is then obvious that

$$
\left\|\bar{y}_{k \Delta}\right\| \leqslant\left\|y_{k \Delta}\right\|, \quad \forall k \geqslant 0 .
$$

Moreover, for any $t \geqslant 0$, let $[t / \Delta]$ be the integer part of $t / \Delta$. Then

$$
\begin{aligned}
\left\|\bar{y}_{t}\right\| & =\left\|\bar{y}_{[t / \Delta] \Delta}\right\| \\
& \leqslant\left\|y_{[t / \Delta] \Delta}\right\| \\
& \leqslant \sup _{-\tau \leqslant s \leqslant t}|y(s)| .
\end{aligned}
$$

This property will be used frequently in what follows, without further explanation.

To illustrate our numerical scheme, as well as to see why we call it the Euler-Maruyama method, let us consider a special SFDE

$$
d x(t)=F\left(D\left(x_{t}\right)\right) d t+G\left(D\left(x_{t}\right)\right) d B(t),
$$

where $F: R^{n} \rightarrow R^{n}, G: R^{n} \rightarrow R^{n \times m}$, and $D$ is a linear operator from $C\left([-\tau, 0] ; R^{n}\right)$ to $R^{n}$ given by

$$
D(\varphi)=\frac{1}{\tau} \int_{-\tau}^{0} \varphi(\theta) d \theta, \quad \varphi \in C\left([-\tau, 0] ; R^{n}\right) ;
$$

that is, $D$ is an average operator. In this case, the discrete approximate solution (2.3) takes the following simple form:

$$
\begin{cases}\bar{y}(k \Delta)=\xi(k \Delta), & -N \leqslant k \leqslant 0, \\ \bar{y}((k+1) \Delta)=\bar{y}(k \Delta)+F\left(D\left(\bar{y}_{k \Delta}\right)\right) \Delta+G\left(D\left(\bar{y}_{k \Delta}\right)\right) \Delta B_{k}, & k \geqslant 0,\end{cases}
$$

where

$$
\begin{aligned}
D\left(\bar{y}_{k \Delta}\right) & =\frac{1}{\tau} \int_{-\tau}^{0} \bar{y}_{k \Delta}(\theta) d \theta \\
& =\frac{1}{\tau} \sum_{i=-N}^{-1} \frac{\Delta}{2}[\bar{y}((k+i) \Delta)+\bar{y}((k+i+1) \Delta)] \\
& =\frac{1}{N}\left(\frac{1}{2} \bar{y}((k-N) \Delta)+\bar{y}((k-N+1) \Delta)+\ldots+\bar{y}((k-1) \Delta)+\frac{1}{2} \bar{y}(k \Delta)\right) .
\end{aligned}
$$

We see clearly from this simple form that the discrete approximate solution (2.3) is a natural generalization of the classical Euler-Maruyama numerical scheme for SDEs, and that is why we call (2.3) the Euler-Maruyama approximate solution.

The primary aim of this paper is to establish the following main result.

THEOREM 2.5. Under Assumptions 2.1-2.3,

$$
\lim _{\Delta \rightarrow 0} E\left(\sup _{0 \leqslant t \leqslant T}|x(t)-y(t)|^{2}\right)=0, \quad \forall T>0 .
$$

The proof of this theorem is very technical, so we present a number of lemmas in Section 3, and then we complete the proof in Section 4. 


\section{Lemmas}

Lemma 3.1. Let Assumption 2.3 hold. Define $\alpha:(0, \tau] \rightarrow R_{+}$by

$$
\alpha(u)=\sup _{t, s \in[-\tau, 0],|t-s|<u} E|\xi(t)-\xi(s)|^{2} .
$$

Then $\alpha$ is nondecreasing and has the property that $\alpha(u) \rightarrow 0$ as $u \rightarrow 0$. Moreover,

$$
E|\xi(t)-\xi(s)|^{2} \leqslant \alpha(t-s), \quad-\tau \leqslant s \leqslant t \leqslant 0 .
$$

Proof. From the definition of $\alpha$ we see clearly that $\alpha$ is nondecreasing and (3.1) holds. We therefore need only to show that $\alpha(u) \rightarrow 0$ as $u \rightarrow 0$. If this is not true, then

$$
\lim _{u \rightarrow 0} \alpha(u)=\varepsilon_{0}>0 .
$$

From the definition of $\alpha$ we observe that for each integer $k \geqslant 1$ we can find a pair of $t_{k}$ and $s_{k}$ in $[-\tau, 0]$ with $\left|t_{k}-s_{k}\right|<1 / k$ for which

$$
E\left|\xi\left(t_{k}\right)-\xi\left(s_{k}\right)\right|^{2} \geqslant \frac{\varepsilon_{0}}{2}
$$

Since $\left\{t_{k}\right\}$ is a sequence in the bounded interval $[-\tau, 0]$, it must have a convergent subsequence. Without any loss of generality, we may assume that $\left\{t_{k}\right\}$ is already a convergent sequence, and that it converges to $\bar{t} \in[-\tau, 0]$. Clearly, $\left\{s_{k}\right\}$ converges to $\bar{t}$ too. Now, by the continuity of $\xi(\cdot)$,

$$
\lim _{k \rightarrow \infty}\left|\xi\left(t_{k}\right)-\xi(\bar{t})\right|^{2}=0, \quad \text { almost surely. }
$$

Moreover,

$$
\begin{aligned}
\left|\xi\left(t_{k}\right)-\xi(\bar{t})\right|^{2} & \leqslant 2\left|\xi\left(t_{k}\right)\right|^{2}+2|\xi(\bar{t})|^{2} \\
& \leqslant 4\|\xi\|^{2}
\end{aligned}
$$

while (by Assumption 2.3 and the Hölder inequality)

$$
E\|\xi\|^{2} \leqslant\left(E\|\xi\|^{p}\right)^{2 / p}<\infty .
$$

We can then apply the dominated convergence theorem to obtain

$$
\lim _{k \rightarrow \infty} E\left|\xi\left(t_{k}\right)-\xi(\bar{t})\right|^{2}=0 \text {. }
$$

Similarly, we can show that

$$
\lim _{k \rightarrow \infty} E\left|\xi\left(s_{k}\right)-\xi(\bar{t})\right|^{2}=0
$$

Consequently, we have

$$
\lim _{k \rightarrow \infty} E\left|\xi\left(t_{k}\right)-\xi\left(s_{k}\right)\right|^{2}=0,
$$

but this is in contradiction to (3.2). We therefore must have

$$
\lim _{u \rightarrow 0} \alpha(u)=0 \text {. }
$$

The proof is therefore complete.

Lemma 3.2. Under Assumptions 2.2 and 2.3,

$$
E\left(\sup _{-\tau \leqslant t \leqslant T}|y(t)|^{p}\right) \leqslant H, \quad \forall T>0,
$$

where $H$ is a positive number dependent only on $\xi, K, p$ and $T$, but independent of $\Delta$. 
Proof. By the Hölder inequality, it is easy to see from (2.7) that

$$
|y(t)|^{p} \leqslant 3^{p-1}\left[|\xi(0)|^{p}+t^{p-1} \int_{0}^{t}\left|f\left(\bar{y}_{s}\right)\right|^{p} d s+\left|\int_{0}^{t} g\left(\bar{y}_{s}\right) d B(s)\right|^{p}\right] .
$$

Hence, for any $t_{1} \in[0, T]$,

$$
\begin{aligned}
E\left(\sup _{0 \leqslant t \leqslant t_{1}}|y(t)|^{p}\right) \leqslant & 3^{p-1}\left[E|\xi(0)|^{p}+T^{p-1} E \int_{0}^{t_{1}}\left|f\left(\bar{y}_{s}\right)\right|^{p} d s\right. \\
& \left.+E\left(\sup _{0 \leqslant t \leqslant t_{1}}\left|\int_{0}^{t} g\left(\bar{y}_{s}\right) d B(s)\right|^{p}\right)\right] .
\end{aligned}
$$

By Assumption 2.2, we compute that

$$
\begin{aligned}
E \int_{0}^{t_{1}}\left|f\left(\bar{y}_{s}\right)\right|^{p} d s & \leqslant 2^{(p-2) / 2} K^{p / 2} E \int_{0}^{t_{1}}\left(1+\|\bar{y}(s)\|^{p}\right) d s \\
& \leqslant 2^{(p-2) / 2} K^{p / 2}\left[T+\int_{0}^{t_{1}} E\left(\sup _{-\tau \leqslant t \leqslant s}|y(t)|^{p}\right) d s\right] .
\end{aligned}
$$

We also compute, using the Burkholder-Davis-Gundy inequality,

$$
\begin{aligned}
E\left(\sup _{0 \leqslant t \leqslant t_{1}}\left|\int_{0}^{t} g\left(\bar{y}_{s}\right) d B(s)\right|^{p}\right) & \leqslant c_{p} E\left(\int_{0}^{t_{1}}\left|g\left(\bar{y}_{s}\right)\right|^{2} d s\right)^{p / 2} \\
& \leqslant c_{p} T^{(p-2) / 2} E \int_{0}^{t_{1}}\left|g\left(\bar{y}_{s}\right)\right|^{p} d s,
\end{aligned}
$$

where $c_{p}$ is a constant dependent only on $p$. In the same way as (3.5) was obtained, we can then show that

$E\left(\sup _{0 \leqslant t \leqslant t_{1}}\left|\int_{0}^{t} g\left(\bar{y}_{s}\right) d B(s)\right|^{p}\right) \leqslant c_{p}(2 T)^{(p-2) / 2} K^{p / 2}\left[T+\int_{0}^{t_{1}} E\left(\sup _{-\tau \leqslant t \leqslant s}|y(t)|^{p}\right) d s\right]$.

Substituting (3.5) and (3.6) into (3.4) yields

$$
E\left(\sup _{0 \leqslant t \leqslant t_{1}}|y(t)|^{p}\right) \leqslant 3^{p-1} E|\xi(0)|^{p}+C_{1}+C_{2} \int_{0}^{t_{1}} E\left(\sup _{-\tau \leqslant t \leqslant s}|y(t)|^{p}\right) d s
$$

where $C_{1}$ and $C_{2}$ are two positive numbers dependent only on $K, p$ and $T$. We then derive the following inequalities:

$$
\begin{aligned}
E\left(\sup _{-\tau \leqslant t \leqslant t_{1}}|y(t)|^{p}\right) & \leqslant E\|\xi\|^{p}+E\left(\sup _{0 \leqslant t \leqslant t_{1}}|y(t)|^{p}\right) \\
& \leqslant\left(1+3^{p-1}\right) E\|\xi\|^{p}+C_{1}+C_{2} \int_{0}^{t_{1}} E\left(\sup _{-\tau \leqslant t \leqslant s}|y(t)|^{p}\right) d s .
\end{aligned}
$$

By the well-known Gronwall inequality we find that

$$
E\left(\sup _{-\tau \leqslant t \leqslant T}|y(t)|^{p}\right) \leqslant\left[\left(1+3^{p-1}\right) E\|\xi\|^{p}+C_{1}\right] e^{C_{2} T},
$$

and hence the required assertion must hold. 
Lemma 3.3. Let Assumptions 2.1-2.3 hold, and let $T>0$. Then there is a nondecreasing function $\beta:(0, \tau] \rightarrow R_{+}$that has the property that $\beta(u)=0$ as $u \rightarrow 0$, such that

$$
E\left|y(s+\theta)-\bar{y}_{s}(\theta)\right|^{2} \leqslant \beta(\Delta), \quad s \in[0, T], \theta \in[-\tau, 0] .
$$

Proof. Fix any $s \in[0, T]$ and $\theta \in[-\tau, 0]$. Let $k_{s}$ and $k_{\theta}$ be the integers for which $s \in\left[k_{s} \Delta,\left(k_{s}+1\right) \Delta\right)$ and $\theta \in\left[k_{\theta} \Delta,\left(k_{\theta}+1\right) \Delta\right]$, respectively. (When $\theta / \Delta$ is an integer, the choice for $k_{\theta}$ may not be unique, but this will not affect the proof below.) Clearly, $0 \leqslant s-k_{s} \Delta<\Delta$ and $0 \leqslant \theta-k_{\theta} \Delta \leqslant \Delta$, so

$$
\begin{aligned}
0 & \leqslant s+\theta-\left(k_{s}+k_{\theta}\right) \Delta \\
& <2 \Delta .
\end{aligned}
$$

Moreover, it follows from (2.4) and (2.6) that

$$
\begin{aligned}
\bar{y}_{s}(\theta) & =\bar{y}_{k_{s} \Delta}(\theta) \\
& =\bar{y}\left(\left(k_{s}+k_{\theta}\right) \Delta\right)+\frac{\theta-k_{\theta} \Delta}{\Delta}\left[\bar{y}\left(\left(k_{s}+k_{\theta}+1\right) \Delta\right)-\bar{y}\left(\left(k_{s}+k_{\theta}\right) \Delta\right)\right] .
\end{aligned}
$$

Hence

$$
\begin{aligned}
E\left|y(s+\theta)-\bar{y}_{s}(\theta)\right|^{2} \leqslant & 2 E\left|y(s+\theta)-\bar{y}\left(\left(k_{s}+k_{\theta}\right) \Delta\right)\right|^{2} \\
& +2 E\left|\bar{y}\left(\left(k_{s}+k_{\theta}+1\right) \Delta\right)-\bar{y}\left(\left(k_{s}+k_{\theta}\right) \Delta\right)\right|^{2} .
\end{aligned}
$$

If $k_{s}+k_{\theta} \leqslant-1$, then, by Lemma 3.1,

$$
E\left|\bar{y}\left(\left(k_{s}+k_{\theta}+1\right) \Delta\right)-\bar{y}\left(\left(k_{s}+k_{\theta}\right) \Delta\right)\right|^{2} \leqslant \alpha(\Delta) .
$$

If $k_{s}+k_{\theta} \geqslant 0$, by Assumption 2.2 and Lemma 3.2 we compute from (2.3) that

$$
\begin{aligned}
E\left|\bar{y}\left(\left(k_{s}+k_{\theta}+1\right) \Delta\right)-\bar{y}\left(\left(k_{s}+k_{\theta}\right) \Delta\right)\right|^{2} \\
\quad=\Delta^{2} E \mid f\left(\bar{y}_{\left.\left(k_{s}+k_{\theta}\right) \Delta\right)\left.\right|^{2}+\Delta E\left|g\left(\bar{y}_{\left(k_{s}+k_{\theta}\right) \Delta}\right)\right|^{2}}\right. \\
\quad \leqslant 2 \Delta K\left(1+E \| \bar{y}_{\left.\left(k_{s}+k_{\theta}\right) \Delta \|^{2}\right)}\right. \\
\quad \leqslant 2 \Delta K\left(1+E\left[\sup _{-\tau \leqslant u \leqslant\left(k_{s}+k_{\theta}\right) \Delta}|y(u)|^{2}\right]\right) \\
\quad \leqslant 2 \Delta K\left\{1+\left(E\left[\sup _{-\tau \leqslant u \leqslant\left(k_{s}+k_{\theta}\right) \Delta}|y(u)|^{p}\right]\right)^{2 / p}\right\} \\
\quad \leqslant 2 K\left(1+H^{2 / p}\right) \Delta,
\end{aligned}
$$

where $H$ is the constant specified in Lemma 3.2. We hence always have

$$
E\left|\bar{y}\left(\left(k_{s}+k_{\theta}+1\right) \Delta\right)-\bar{y}\left(\left(k_{s}+k_{\theta}\right) \Delta\right)\right|^{2} \leqslant 2 K\left(1+H^{2 / p}\right) \Delta+\alpha(\Delta) .
$$

Using this bound in (3.11) gives

$$
E\left|y(s+\theta)-\bar{y}_{s}(\theta)\right|^{2} \leqslant 2 E\left|y(s+\theta)-\bar{y}\left(\left(k_{s}+k_{\theta}\right) \Delta\right)\right|^{2}+4 K\left(1+H^{2 / p}\right) \Delta+2 \alpha(\Delta) .
$$

To bound the first term on the right-hand side, let us discuss the following four possible cases.

Case 1: $k_{s}+k_{\theta} \geqslant 0$. It follows from (2.8) that

$$
y(s+\theta)-\bar{y}\left(\left(k_{s}+k_{\theta}\right) \Delta\right)=\int_{\left(k_{s}+k_{\theta}\right) \Delta}^{s+\theta} f\left(\bar{y}_{r}\right) d r+\int_{\left(k_{s}+k_{\theta}\right) \Delta}^{s+\theta} g\left(\bar{y}_{r}\right) d B(r) .
$$


By Assumption 2.2 and Lemma 3.2, we compute that

$$
\begin{aligned}
E\left|y(s+\theta)-\bar{y}\left(\left(k_{s}+k_{\theta}\right) \Delta\right)\right|^{2} & \leqslant 2\left[2 \Delta E \int_{\left(k_{s}+k_{\theta}\right) \Delta}^{s+\theta}\left|f\left(\bar{y}_{r}\right)\right|^{2} d r+E \int_{\left(k_{s}+k_{\theta}\right) \Delta}^{s+\theta}\left|g\left(\bar{y}_{r}\right)\right|^{2} d r\right] \\
& \left.\leqslant 6 K E \int_{\left(k_{s}+k_{\theta}\right) \Delta}^{s+\theta}\left(1+\| \bar{y}_{r}\right) \|^{2}\right) d r \\
& \leqslant 6 K \int_{\left(k_{s}+k_{\theta}\right) \Delta}^{s+\theta}\left(1+E\left[\sup _{-\tau \leqslant u \leqslant r}|y(u)|^{2}\right]\right) d r \\
& \leqslant 6 K \int_{\left(k_{s}+k_{\theta}\right) \Delta}^{s+\theta}\left\{1+\left(E\left[\sup _{-\tau \leqslant u \leqslant r}|y(u)|^{p}\right]\right)^{2 / p}\right\} d r \\
& \leqslant 12 K\left(1+H^{2 / p}\right) \Delta .
\end{aligned}
$$

Case 2: $k_{s}+k_{\theta}=-1$ and $\Delta<s+\theta-\left(k_{s}+k_{\theta}\right) \Delta<2 \Delta . \quad$ In this case,

$$
\begin{aligned}
0 & \leqslant \Delta+\left(k_{s}+k_{\theta}\right) \Delta \\
& <s+\theta \\
& <2 \Delta+\left(k_{s}+k_{\theta}\right) \Delta \\
& =\Delta .
\end{aligned}
$$

So

$$
\begin{aligned}
E\left|y(s+\theta)-\bar{y}\left(\left(k_{s}+k_{\theta}\right) \Delta\right)\right|^{2} & =E|y(s+\theta)-\bar{y}(-\Delta)|^{2} \\
& \leqslant E|y(s+\theta)-\xi(0)|^{2}+2 E|\xi(0)-\xi(-\Delta)|^{2} .
\end{aligned}
$$

It can be shown in the same way as in Case 1 that

$$
E|y(s+\theta)-\xi(0)|^{2} \leqslant 4 K\left(1+H^{2 / p}\right) \Delta,
$$

while by Lemma 3.1,

$$
E|\xi(0)-\xi(-\Delta)|^{2} \leqslant \alpha(\Delta)
$$

We therefore see that

$$
E\left|y(s+\theta)-\bar{y}\left(\left(k_{s}+k_{\theta}\right) \Delta\right)\right|^{2} \leqslant 8 K\left(1+H^{2 / p}\right) \Delta+2 \alpha(\Delta) .
$$

Case 3: $k_{s}+k_{\theta}=-1$ and $0 \leqslant s+\theta-\left(k_{s}+k_{\theta}\right) \Delta \leqslant \Delta . \quad$ In this case,

$$
\begin{aligned}
-\Delta & \leqslant\left(k_{s}+k_{\theta}\right) \Delta \\
& <s+\theta \\
& <\Delta+\left(k_{s}+k_{\theta}\right) \Delta \\
& =0 .
\end{aligned}
$$

So

$$
\begin{aligned}
E\left|y(s+\theta)-\bar{y}\left(\left(k_{s}+k_{\theta}\right) \Delta\right)\right|^{2} & =E|\xi(s+\theta)-\bar{y}(-\Delta)|^{2} \\
& =E|\xi(s+\theta)-\xi(-\Delta)|^{2} .
\end{aligned}
$$

By Lemma 3.1, we then have

$$
E\left|y(s+\theta)-\bar{y}_{s}(\theta)\right|^{2} \leqslant \alpha(\Delta) .
$$


Case 4: $k_{s}+k_{\theta} \leqslant-2$. In this case we have $s+\theta \leqslant 0$. So

$$
E\left|y(s+\theta)-\bar{y}\left(\left(k_{s}+k_{\theta}\right) \Delta\right)\right|^{2}=E\left|\xi(s+\theta)-\xi\left(\left(k_{s}+k_{\theta}\right) \Delta\right)\right|^{2} .
$$

By Lemma 3.1 and (3.10), we then have

$$
E\left|y(s+\theta)-\bar{y}\left(\left(k_{s}+k_{\theta}\right) \Delta\right)\right|^{2} \leqslant \alpha(2 \Delta) .
$$

Combining the four cases above together, we can conclude that we always have

$$
E\left|y(s+\theta)-\bar{y}\left(\left(k_{s}+k_{\theta}\right) \Delta\right)\right|^{2} \leqslant 12 K\left(1+H^{2 / p}\right) \Delta+2 \alpha(2 \Delta) .
$$

Now, we define $\beta:(0, \tau] \rightarrow R_{+}$by

$$
\beta(u)=28 K\left(1+H^{2 / p}\right) u+6 \alpha(2 u) .
$$

Clearly, $\beta$ is nondecreasing. Moreover, it follows from (3.12) and (3.17) that

$$
E\left|y(s+\theta)-\bar{y}_{s}(\theta)\right|^{2} \leqslant \beta(\Delta),
$$

which is the required assertion. The proof is complete.

\section{Proof of Theorem 2.5}

Let us now begin to prove Theorem 2.5. We first note from Theorem 2.4 and Lemma 3.2 that there is a positive constant $\bar{H}$ such that

$$
E\left(\sup _{-\tau \leqslant t \leqslant T}|x(t)|^{p}\right) \vee E\left(\sup _{-\tau \leqslant t \leqslant T}|y(t)|^{p}\right) \leqslant \bar{H} .
$$

Let $j$ be a sufficiently large integer. Define the stopping times

$$
u_{j}:=\inf \left\{t \geqslant 0:\left\|x_{t}\right\| \geqslant j\right\}, \quad v_{i}:=\inf \left\{t \geqslant 0:\left\|y_{t}\right\| \geqslant j\right\}, \quad \rho_{j}:=u_{j} \wedge v_{j},
$$

where we set $\inf \emptyset=\infty$ as usual. Let

$$
e(t):=x(t)-y(t) .
$$

Obviously,

$$
E\left[\sup _{0 \leqslant t \leqslant T}|e(t)|^{2}\right]=E\left[\sup _{0 \leqslant t \leqslant T}|e(t)|^{2} \mathbf{1}_{\left\{u_{j}>T, v_{j}>T\right\}}\right]+E\left[\sup _{0 \leqslant t \leqslant T}|e(t)|^{2} \mathbf{1}_{\left\{u_{j} \leqslant T \text { or } v_{j} \leqslant T\right\}}\right] .
$$

Recall the following elementary inequality:

$$
a^{\gamma} b^{1-\gamma} \leqslant \gamma a+(1-\gamma) b, \quad \forall a, b>0, \gamma \in[0,1] .
$$

We thus have, for any $\delta>0$,

$$
\begin{aligned}
& E\left[\sup _{0 \leqslant t \leqslant T}|e(t)|^{2} \mathbf{1}_{\left\{u_{j} \leqslant T \text { or } v_{j} \leqslant T\right\}}\right] \\
& \quad=E\left[\left(\delta \sup _{0 \leqslant t \leqslant T}|e(t)|^{p}\right)^{2 / p}\left(\delta^{-2 /(p-2)} \mathbf{1}_{\left\{u_{j} \leqslant T \text { or } v_{j} \leqslant T\right\}}\right)^{(p-2) / p}\right] \\
& \quad \leqslant \frac{2 \delta}{p} E\left[\sup _{0 \leqslant t \leqslant T}|e(t)|^{p}\right]+\frac{p-2}{p \delta^{2 /(p-2)}} P\left(u_{j} \leqslant T \text { or } v_{j} \leqslant T\right) .
\end{aligned}
$$


Hence

$$
\begin{aligned}
E\left[\sup _{0 \leqslant t \leqslant T}|e(t)|^{2}\right] \leqslant & E\left[\sup _{0 \leqslant t \leqslant T}|e(t)|^{2} \mathbf{1}_{\left\{\rho_{j}>T\right\}}\right]+\frac{2 \delta}{p} E\left[\sup _{0 \leqslant t \leqslant T}|e(t)|^{p}\right] \\
& +\frac{p-2}{p \delta^{2 /(p-2)}} P\left(u_{j} \leqslant T \text { or } v_{j} \leqslant T\right) .
\end{aligned}
$$

Now,

$$
\begin{aligned}
P\left(u_{j} \leqslant T\right) & =E\left[\mathbf{1}_{\left\{u_{j} \leqslant T\right\}} \frac{\left\|x_{u_{j}}\right\|^{p}}{j^{p}}\right] \\
& \leqslant \frac{1}{j^{p}} E\left[\sup _{-\tau \leqslant t \leqslant T}|x(t)|^{p}\right] \\
& \leqslant \frac{\bar{H}}{j^{p}}
\end{aligned}
$$

using (4.1). Similarly, we have

$$
P\left(v_{j} \leqslant T\right) \leqslant \frac{\bar{H}}{j^{p}}
$$

Thus

$$
\begin{aligned}
P\left(u_{j} \leqslant T \text { or } v_{j} \leqslant T\right) & \leqslant P\left(u_{j} \leqslant T\right)+P\left(v_{j} \leqslant T\right) \\
& \leqslant \frac{2 \bar{H}}{j^{p}} .
\end{aligned}
$$

We also have

$$
\begin{aligned}
E\left[\sup _{0 \leqslant t \leqslant T}|e(t)|^{p}\right] & \leqslant 2^{p-1} E\left[\sup _{0 \leqslant t \leqslant T}\left(|x(t)|^{p}+|y(t)|^{p}\right)\right] \\
& \leqslant 2^{p} \bar{H}
\end{aligned}
$$

Moreover,

$$
\begin{aligned}
E\left[\sup _{0 \leqslant t \leqslant T}|e(t)|^{2} \mathbf{1}_{\left\{\rho_{j}>T\right\}}\right] & =E\left[\sup _{0 \leqslant t \leqslant T}\left|e\left(t \wedge \rho_{j}\right)\right|^{2} \mathbf{1}_{\left\{\rho_{j}>T\right\}}\right] \\
& \leqslant E\left[\sup _{0 \leqslant t \leqslant T}\left|e\left(t \wedge \rho_{j}\right)\right|^{2}\right] .
\end{aligned}
$$

Using these bounds in (4.2) yields

$$
E\left[\sup _{0 \leqslant t \leqslant T}|e(t)|^{2}\right] \leqslant E\left[\sup _{0 \leqslant t \leqslant T}\left|e\left(t \wedge \rho_{j}\right)\right|^{2}\right]+\frac{2^{p+1} \delta \bar{H}}{p}+\frac{(p-2) 2 \bar{H}}{p \delta^{2 /(p-2)} j^{p}} .
$$

Now

$$
\begin{aligned}
\left|e\left(t \wedge \rho_{j}\right)\right|^{2} & =\left|x\left(t \wedge \rho_{j}\right)-y\left(t \wedge \rho_{j}\right)\right|^{2} \\
& =\left|\int_{0}^{t \wedge \rho_{j}}\left[f\left(x_{s}\right)-f\left(\bar{y}_{s}\right)\right] d s+\int_{0}^{t \wedge \rho_{j}}\left[g\left(x_{s}\right)-g\left(\bar{y}_{s}\right)\right] d B(s)\right|^{2} \\
& \leqslant 2\left[T \int_{0}^{t \wedge \rho_{j}}\left|f\left(x_{s}\right)-f\left(\bar{y}_{s}\right)\right|^{2} d s+\left|\int_{0}^{t \wedge \rho_{j}}\left[g\left(x_{s}\right)-g\left(\bar{y}_{s}\right)\right] d B(s)\right|^{2}\right] .
\end{aligned}
$$




\section{Stochastic functional differential equations}

By the Doob martingale inequality we have, for any $t_{1} \leqslant T$,

$$
\begin{aligned}
& E\left[\sup _{0 \leqslant t \leqslant t_{1}}\left|e\left(t \wedge \rho_{j}\right)\right|^{2}\right] \\
& \quad \leqslant 2\left[T E \int_{0}^{t_{1} \wedge \rho_{j}}\left|f\left(x_{s}\right)-f\left(\bar{y}_{s}\right)\right|^{2} d s+4 \int_{0}^{t_{1} \wedge \rho_{j}}\left|g\left(x_{s}\right)-g\left(\bar{y}_{s}\right)\right|^{2} d s\right] \\
& \quad=4(T+4) E \int_{0}^{t_{1} \wedge \rho_{j}}\left[\left|f\left(x_{s}\right)-f\left(\bar{y}_{s}\right)\right|^{2} \vee\left|g\left(x_{s}\right)-g\left(\bar{y}_{s}\right)\right|^{2}\right] d s .
\end{aligned}
$$

But, by Assumption 2.1, we derive that, for $s \in\left(0, t_{1} \wedge \rho_{j}\right]$,

$$
\begin{aligned}
& \left|f\left(x_{s}\right)-f\left(\bar{y}_{s}\right)\right|^{2} \\
& \quad \leqslant 2\left|f\left(x_{s}\right)-f\left(y_{s}\right)\right|^{2}+2\left|f\left(y_{s}\right)-f\left(\bar{y}_{s}\right)\right|^{2} \\
& \quad \leqslant 2 \int_{-\tau}^{0}|x(s+\theta)-y(s+\theta)|^{2} d \mu_{j}(\theta)+2 \int_{-\tau}^{0}\left|y(s+\theta)-\bar{y}_{s}(\theta)\right|^{2} d \mu_{j}(\theta) \\
& \quad \leqslant 2 \int_{-\tau}^{0}\left[\sup _{-\tau \leqslant \theta \leqslant 0}|x(s+\theta)-y(s+\theta)|^{2}\right] d \mu_{j}(\theta)+2 \int_{-\tau}^{0}\left|y(s+\theta)-\bar{y}_{s}(\theta)\right|^{2} d \mu_{j}(\theta) \\
& \quad \leqslant 2\left(\mu_{j}(0)-\mu_{j}(-\tau)\right)\left[\sup _{0 \leqslant t \leqslant s}|x(t)-y(t)|^{2}\right]+2 \int_{-\tau}^{0}\left|y(s+\theta)-\bar{y}_{s}(\theta)\right|^{2} d \mu_{j}(\theta) .
\end{aligned}
$$

A similar result can be obtained for $\left|g\left(x_{S}\right)-g\left(\bar{y}_{S}\right)\right|^{2}$, so that

$$
\begin{aligned}
E\left[\sup _{0 \leqslant t \leqslant t_{1}}\left|e\left(t \wedge \rho_{j}\right)\right|^{2}\right] \leqslant & 8(T+4)\left(\mu_{j}(0)-\mu_{j}(-\tau)\right) E \int_{0}^{t_{1} \wedge \rho_{j}}\left[\sup _{0 \leqslant t \leqslant s}|e(s)|^{2}\right] d s \\
& +8(T+4) E \int_{0}^{t_{1} \wedge \rho_{j}}\left[\int_{-\tau}^{0}\left|y(s+\theta)-\bar{y}_{s}(\theta)\right|^{2} d \mu_{j}(\theta)\right] d s \\
\leqslant & 8(T+4)\left(\mu_{j}(0)-\mu_{j}(-\tau)\right) \int_{0}^{t_{1}} E\left[\sup _{0 \leqslant t \leqslant s}\left|e\left(s \wedge \rho_{j}\right)\right|^{2}\right] d s \\
& +8(T+4) \int_{0}^{T}\left[\int_{-\tau}^{0} E\left|y(s+\theta)-\bar{y}_{s}(\theta)\right|^{2} d \mu_{j}(\theta)\right] d s .
\end{aligned}
$$

By Lemma 3.3 we therefore find that

$$
\begin{aligned}
E\left[\sup _{0 \leqslant t \leqslant t_{1}}\left|e\left(t \wedge \rho_{j}\right)\right|^{2}\right] \leqslant & 8(T+4)\left(\mu_{j}(0)-\mu_{j}(-\tau)\right) \int_{0}^{t_{1}} E\left[\sup _{0 \leqslant t \leqslant s}\left|e\left(s \wedge \rho_{j}\right)\right|^{2}\right] d s \\
& +8 T(T+4)\left(\mu_{j}(0)-\mu_{j}(-\tau)\right) \beta(\Delta) .
\end{aligned}
$$

The Gronwall inequality implies that

$$
E\left[\sup _{0 \leqslant t \leqslant T}\left|e\left(t \wedge \rho_{j}\right)\right|^{2}\right] \leqslant C_{j} \beta(\Delta),
$$

where

$$
C_{j}=8 T(T+4)\left(\mu_{j}(0)-\mu_{j}(-\tau)\right) \exp \left[8 T(T+4)\left(\mu_{j}(0)-\mu_{j}(-\tau)\right)\right] .
$$

Substituting this into (4.3) gives

$$
E\left[\sup _{0 \leqslant t \leqslant T}|e(t)|^{2}\right] \leqslant C_{j} \beta(\Delta)+\frac{2^{p+1} \delta \bar{H}}{p}+\frac{(p-2) 2 \bar{H}}{p \delta^{2 /(p-2) j^{p}}} .
$$


Given any $\varepsilon>0$, we can now choose $\delta$ sufficiently small for $\left(2^{p+1} \delta \bar{H}\right) / p<\varepsilon / 3$, then choose $j$ sufficiently large for

$$
\frac{(p-2) 2 \bar{H}}{p \delta^{2 /(p-2)} j^{p}}<\frac{\varepsilon}{3}
$$

and finally choose $\Delta$ so that

$$
C_{j} \beta(\Delta)<\frac{\varepsilon}{3}
$$

Thus, in (4.6), $E\left[\sup _{0 \leqslant t \leqslant T}|e(t)|^{2}\right]<\varepsilon$, as required. The proof of Theorem 2.5 is therefore complete.

\section{Order of convergence under the global Lipschitz condition}

Under the general conditions (namely the local Lipschitz condition and the linear growth condition) that are normally imposed for the existence and uniqueness of the solution, Theorem 2.5 shows the strong convergence of the Euler-Maruyama approximate solutions to the true solution. However, this theorem does not give the order of the convergence. In this section we reveal the order of the convergence, but here we need to replace the local Lipschitz condition by the global Lipschitz condition. To be more precise, we state the assumption as follows.

Assumption 5.1 (THE GLOBAL LiPSCHITZ CONDITION). There is a right-continuous nondecreasing function $\mu:[-\tau, 0] \rightarrow R_{+}$such that

$$
|f(\varphi)-f(\psi)|^{2} \vee|g(\varphi)-g(\psi)|^{2} \leqslant \int_{-\tau}^{0}|\varphi(\theta)-\psi(\theta)|^{2} d \mu(\theta)
$$

for all $\varphi, \psi \in C\left([-\tau, 0] ; R^{n}\right)$.

It is easy to see from this global Lipschitz condition that for any $\varphi \in C\left([-\tau, 0] ; R^{n}\right)$,

$$
|f(\varphi)|^{2} \vee|g(\varphi)|^{2} \leqslant 2\left(|f(0)|^{2} \vee|g(0)|^{2}\right)+2(\mu(0)-\mu(-\tau))\|\varphi\|^{2} .
$$

That is, the global Lipschitz condition implies the linear growth condition (Assumption 2.2) with the growth coefficient

$$
K=2\left[\left(|f(0)|^{2} \vee|g(0)|^{2}\right) \vee(\mu(0)-\mu(-\tau))\right] .
$$

In addition, we need to slightly strengthen Assumption 2.3 on the initial data.

Assumption 5.2. Assume that $\xi \in L_{\widetilde{F}_{0}}^{p}\left([-\tau, 0] ; R^{n}\right)$ for some $p>2$; moreover, there is a positive constant $\lambda$ such that

$$
E|\xi(t)-\xi(s)|^{2} \leqslant \lambda(t-s), \quad-\tau \leqslant s \leqslant t \leqslant 0 .
$$

We can now state another theorem, which reveals the order of the convergence.

THEOREM 5.3. Under Assumptions 5.1 and 5.2,

$$
E\left(\sup _{0 \leqslant t \leqslant T}|x(t)-y(t)|^{2}\right)=O(\Delta), \quad \forall T>0 .
$$

Proof. First of all, we note that Lemma 3.1 is now replaced by Assumption 5.2, and the function $\alpha(\cdot)$ there takes the simple linear form $\alpha(u)=\lambda u$. Moreover, Lemmas 3.2 and 3.3 
hold with $K$ defined by (5.1), while the function $\beta(\cdot)$ in Lemma 3.3 is now a linear function, namely

$$
\beta(u)=4\left(7 K\left(1+H^{2 / p}\right)+3 \lambda\right) u .
$$

So the proof of Theorem 2.5 still works, except the local Lipschitz condition has now been replaced by the global one; in other words, $\mu_{j}$ is replaced by $\mu$. As a result, the constant $C_{j}$ in (4.4) becomes

$$
C=8 T(T+4)(\mu(0)-\mu(-\tau)) \exp [8 T(T+4)(\mu(0)-\mu(-\tau))],
$$

which is independent of $j$. So inequality (4.4) can be written as

$$
E\left[\sup _{0 \leqslant t \leqslant T}|e(t)|^{2}\right] \leqslant C \beta(\Delta)+\frac{2^{p+1} \delta \bar{H}}{p}+\frac{(p-2) 2 \bar{H}}{p \delta^{2 /(p-2) j^{p}} .}
$$

Since this holds for arbitrary $\delta>0$ and $j>1$, we may let $j \rightarrow \infty$ and $\delta \rightarrow 0$, in order to obtain

$$
E\left[\sup _{0 \leqslant t \leqslant T}|e(t)|^{2}\right] \leqslant C \beta(\Delta)=4 C\left(3 K\left(1+H^{2 / p}\right)+\lambda\right) \Delta,
$$

as required. The proof is complete.

\section{A generalized result}

We observe from the proof of Theorem 2.5 that it is the $p$ th moment bound (4.1) that plays an important role, while the linear growth condition (namely Assumption 2.2) is mainly used to guarantee (4.1). We therefore wonder whether Theorem 2.5 would still hold if Assumption 2.2 were replaced by (4.1). The main aim of this section is to give a positive answer to this question. Let us state (4.1) as an assumption.

Assumption 6.1. For some $p>2$, there is a positive constant $\bar{H}$, which may depend on $\xi, p$ and $T$, but is independent of $\Delta$, such that

$$
E\left(\sup _{-\tau \leqslant t \leqslant T}|x(t)|^{p}\right) \vee E\left(\sup _{-\tau \leqslant t \leqslant T}|y(t)|^{p}\right) \leqslant \bar{H} .
$$

Our generalized result can now be stated as follows.

THEOREM 6.2. Under Assumptions 2.1, 2.3 and 6.1,

$$
\lim _{\Delta \rightarrow 0} E\left(\sup _{0 \leqslant t \leqslant T}|x(t)-y(t)|^{2}\right)=0, \quad \forall T>0 .
$$

Proof. We use the same notations as in the proof of Theorem 2.5, and we note that everything up to inequality (4.4) still holds. However, we observe that we derived (4.5) from (4.4) in order to apply Lemma 3.3, which requires Assumption 2.2. It is there that we need a much more careful consideration, and we use a truncation technique to overcome the difficulty.

Let $j$ be a positive integer, and let $S_{j}=\left\{x \in R^{n}:|x| \leqslant j\right\}$. Define the projection $\pi_{j}: R^{n} \rightarrow S_{j}$ by

$$
\pi_{j}(x)=\frac{j \wedge|x|}{|x|} x
$$

where we set $\pi_{j}(0)=0$ as usual. 
It is easy to see that

$$
\left|\pi_{j}(x)-\pi_{j}(y)\right| \leqslant|x-y|, \quad \forall x, y \in R^{n} .
$$

Define the operator $\bar{\pi}_{j}: C\left([-\tau, 0] ; R^{n}\right) \rightarrow C\left([-\tau, 0] ; R^{n}\right)$ by

$$
\bar{\pi}_{j}(\varphi)=\left\{\pi_{j}(\varphi(\theta)):-\tau \leqslant \theta \leqslant 0\right\} .
$$

Clearly,

$$
\left\|\bar{\pi}_{j}(\varphi)\right\| \leqslant j, \quad \forall \varphi \in C\left([-\tau, 0] ; R^{n}\right) .
$$

Define the truncation functions $f_{j}: C\left([-\tau, 0] ; R^{n}\right) \rightarrow R^{n}$ and $g_{j}: C\left([-\tau, 0] ; R^{n}\right) \rightarrow$ $R^{n \times m}$ by

$$
f_{j}(\varphi)=f\left(\bar{\pi}_{j}(\varphi)\right) \quad \text { and } \quad g_{j}(\varphi)=g\left(\bar{\pi}_{j}(\varphi)\right),
$$

respectively. By Assumption 2.1, we compute that for any $\varphi, \psi \in C\left([-\tau, 0] ; R^{n}\right)$,

$$
\begin{aligned}
\mid f_{j}(\varphi) & -\left.f_{j}(\psi)\right|^{2} \vee\left|g_{j}(\varphi)-g_{j}(\psi)\right|^{2} \\
= & \left|f\left(\bar{\pi}_{j}(\varphi)\right)-f\left(\bar{\pi}_{j}(\psi)\right)\right|^{2} \vee\left|g\left(\bar{\pi}_{j}(\varphi)\right)-g\left(\bar{\pi}_{j}(\psi)\right)\right|^{2} \\
& \leqslant \int_{-\tau}^{0}\left|\pi_{j}(\varphi(\theta))-\pi_{j}(\psi(\theta))\right|^{2} d \mu_{j}(\theta) \\
& \leqslant \int_{-\tau}^{0}|\varphi(\theta)-\psi(\theta)|^{2} d \mu_{j}(\theta),
\end{aligned}
$$

and

$$
\left|f_{j}(\varphi)\right|^{2} \vee\left|g_{j}(\varphi)\right|^{2} \leqslant K\left(1+\|\varphi\|^{2}\right),
$$

where

$$
K=K(j)=2\left[\left(|f(0)|^{2} \vee|g(0)|^{2}\right) \vee\left(\mu_{j}(0)-\mu_{j}(-\tau)\right)\right] .
$$

In other words, $f_{j}$ and $g_{j}$ satisfy both the global Lipschitz condition and the linear growth condition. Consider the SFDE

$$
d x^{j}=f_{j}\left(x_{t}^{j}\right) d t+g_{j}\left(x_{t}^{j}\right) d B(t), \quad t \geqslant 0,
$$

with initial data $x_{0}^{j}=\xi$. Applying the Euler-Maruyama method to this SFDE, we can define: the discrete approximate solution $\bar{y}^{j}(k \Delta)$ for $k \geqslant-N$, the $C\left([-\tau, 0] ; R^{n}\right)$-value step process

$$
\bar{y}_{t}^{j}=\sum_{k=0}^{\infty} \bar{y}_{k \Delta}^{j} 1_{[k \Delta,(k+1) \Delta)}(t), \quad t \geqslant 0,
$$

and then the continuous Euler-Maruyama approximate solution

$$
y^{j}(t)= \begin{cases}\xi(t), & -\tau \leqslant t \leqslant 0, \\ \xi(0)+\int_{0}^{t} f_{j}\left(\bar{y}_{s}^{j}\right) d s+\int_{0}^{t} g_{j}\left(\bar{y}_{s}^{j}\right) d B(s), \quad t \geqslant 0 .\end{cases}
$$

Applying Lemma 3.3 to $(6.5)$, we see that there is a nondecreasing function $\beta_{j}:(0, \tau] \rightarrow$ $R_{+}$, which has the property that $\beta_{j}(u)=0$ as $u \rightarrow 0$, such that

$$
E\left|y^{j}(s+\theta)-\bar{y}_{s}^{j}(\theta)\right|^{2} \leqslant \beta_{j}(\Delta), \quad s \in[0, T], \theta \in[-\tau, 0] .
$$


Moreover, by the definition of $\rho_{j}$, it is easy to observe that for $s \in\left(0, t_{1} \wedge \rho_{j}\right]$, we have $\bar{y}_{s}=\bar{y}_{s}^{j}$ and

$$
\begin{aligned}
y(s) & =\xi(0)+\int_{0}^{s} f\left(\bar{y}_{r}\right) d r+\int_{0}^{s} g\left(\bar{y}_{r}\right) d B(r) \\
& =\xi(0)+\int_{0}^{s} f_{j}\left(\bar{y}_{r}^{j}\right) d r+\int_{0}^{s} g_{j}\left(\bar{y}_{r}^{j}\right) d B(r) \\
& =y^{j}(s) .
\end{aligned}
$$

By (6.6), we then derive from (4.4) that

$$
\begin{aligned}
E\left[\sup _{0 \leqslant t \leqslant t_{1}}\left|e\left(t \wedge \rho_{j}\right)\right|^{2}\right] \leqslant & 8(T+4)\left(\mu_{j}(0)-\mu_{j}(-\tau)\right) E \int_{0}^{t_{1} \wedge \rho_{j}}\left[\sup _{0 \leqslant t \leqslant s}|e(s)|^{2}\right] d s \\
& +8(T+4) E \int_{0}^{t_{1} \wedge \rho_{j}}\left[\int_{-\tau}^{0}\left|y^{j}(s+\theta)-\bar{y}_{s}^{j}(\theta)\right|^{2} d \mu_{j}(\theta)\right] d s \\
\leqslant & 8(T+4)\left(\mu_{j}(0)-\mu_{j}(-\tau)\right) E \int_{0}^{t_{1}}\left[\sup _{0 \leqslant t \leqslant s}\left|e\left(s \wedge \rho_{j}\right)\right|^{2}\right] d s \\
& +8(T+4) \int_{0}^{T}\left[\int_{-\tau}^{0} E\left|y^{j}(s+\theta)-\bar{y}_{s}^{j}(\theta)\right|^{2} d \mu_{j}(\theta)\right] d s \\
\leqslant & 8(T+4)\left(\mu_{j}(0)-\mu_{j}(-\tau)\right) \int_{0}^{t_{1}} E\left[\sup _{0 \leqslant t \leqslant s}\left|e\left(s \wedge \rho_{j}\right)\right|^{2}\right] d s \\
& +8 T(T+4)\left(\mu_{j}(0)-\mu_{j}(-\tau)\right) \beta_{j}(\Delta) .
\end{aligned}
$$

The Gronwall inequality implies that

$$
E\left[\sup _{0 \leqslant t \leqslant T}\left|e\left(t \wedge \rho_{j}\right)\right|^{2}\right] \leqslant C_{j} \beta_{j}(\Delta),
$$

where $C_{j}$ is as defined in the proof of Theorem 2.5. The remainder of the proof is the same as before, so now the proof is complete.

\section{A simpler scheme}

In this section we establish a simpler version of the Euler-Maruyama numerical scheme than the one that we introduced in Section 2. To motivate the new scheme, let us consider a special SFDE

$$
d x(t)=F\left(D\left(x_{t}\right)\right) d t+G\left(D\left(x_{t}\right)\right) d B(t),
$$

where $F: R^{n} \rightarrow R^{n}, G: R^{n} \rightarrow R^{n \times m}$ and $D$ is a nonlinear operator from $C\left([-\tau, 0] ; R^{n}\right)$ to $R^{n}$ given by

$$
D(\varphi)=\int_{-\tau}^{0} \kappa(\varphi(\theta)) d \theta, \quad \varphi \in C\left([-\tau, 0] ; R^{n}\right),
$$

in which $\kappa$ is a continuous function from $R^{n}$ to itself. In this case, the discrete approximate solution (2.3) takes the following simple form:

$$
\begin{cases}\bar{y}(k \Delta)=\xi(k \Delta), & -N \leqslant k \leqslant 0, \\ \bar{y}((k+1) \Delta)=\bar{y}(k \Delta)+F\left(D\left(\bar{y}_{k \Delta}\right)\right) \Delta+G\left(D\left(\bar{y}_{k \Delta}\right)\right) \Delta B_{k}, \quad k \geqslant 0,\end{cases}
$$


where

$$
D\left(\bar{y}_{k \Delta}\right)=\int_{-\tau}^{0} \kappa\left(\bar{y}_{k \Delta}(\theta)\right) d \theta .
$$

Since $\kappa\left(\bar{y}_{k \Delta}(\theta)\right)$ is continuous in $\theta \in[-\tau, 0]$, the above integration may be approximated by the following simple sum:

$$
\sum_{i=-N}^{-1} \kappa(\bar{y}((k+i) \Delta)) \Delta
$$

This is equivalent to saying that $\bar{y}_{k \Delta}$ is defined as a step-function-valued random variable as follows:

$$
\left\{\begin{array}{l}
\bar{y}_{k \Delta}(\theta)=\bar{y}((k-i) \Delta) \text { for }-i \Delta \leqslant \theta<(-i+1) \Delta, \quad i=N,(N-1), \ldots, 1, \\
\bar{y}_{k \Delta}(0)=\bar{y}(k \Delta),
\end{array}\right.
$$

instead of a $C\left([-\tau, 0] ; R^{n}\right)$-valued random variable as defined by (2.4). The question is: should $\bar{y}_{k \Delta}$ be defined by (7.3) instead of (2.4), and will the numerical solution (7.2) still converge to the true solution of equation (7.1)? There seems no reason why it should not work. However, when we think more generally to see whether Theorems 2.5 and 6.2 still hold in this way, we first face a problem: both coefficients $f$ and $g$ of equation (2.1) are defined only on $C\left([-\tau, 0] ; R^{n}\right)$. If we define $\bar{y}_{k \Delta}$ by (7.3), then $f\left(\bar{y}_{k \Delta}\right)$ and $g\left(\bar{y}_{k \Delta}\right)$ may not be well defined, and hence the discrete approximate solution (2.3) may not make sense at all, let alone converge to the true solution.

However, if we have another look at equation (7.2), we find there is no such problem for equation (7.2), since the operator $D$ can be naturally extended to step functions. This gives us an idea that we need to extend the domain of $f$ and $g$ to include at least step functions as well, in order to find a simpler numerical scheme. Now that the domain needs to include continuous and step functions, as well as their linear combinations, it is better to work on the space of cadlag (that is, right continuous and left limit) functions from the stochastic analysis point of view. We therefore denote by $C_{a}\left([-\tau, 0] ; R^{n}\right)$ the family of cadlag functions $\varphi$ from $[-\tau, 0]$ to $R^{n}$ with the norm $\|\varphi\|=\sup _{-\tau \leqslant \theta \leqslant 0}|\varphi(\theta)|$. Clearly, $C\left([-\tau, 0] ; R^{n}\right) \subset C_{a}\left([-\tau, 0] ; R^{n}\right)$. From now on, we assume that

$$
f: C_{a}\left([-\tau, 0] ; R^{n}\right) \rightarrow R^{n} \quad \text { and } \quad g: C_{a}\left([-\tau, 0] ; R^{n}\right) \rightarrow R^{n \times m} .
$$

Assumptions 2.1 and 2.2 need to be changed accordingly, so we state the new assumptions.

AsSUMPTION 7.1 (THE LOCAL LiPSCHITZ CONDITION). For each integer $j \geqslant 1$, there is a right-continuous nondecreasing function $\mu_{j}:[-\tau, 0] \rightarrow R_{+}$such that

$$
|f(\varphi)-f(\psi)|^{2} \vee|g(\varphi)-g(\psi)|^{2} \leqslant \int_{-\tau}^{0}|\varphi(\theta)-\psi(\theta)|^{2} d \mu_{j}(\theta)
$$

for those $\varphi, \psi \in C_{a}\left([-\tau, 0] ; R^{n}\right)$ with $\|\varphi\| \vee\|\psi\| \leqslant j$.

AsSUMPTION 7.2 (THE LINEAR GROWTH CONDITION). There is a constant $K>0$ such that

$$
|f(\varphi)|^{2} \vee|g(\varphi)|^{2} \leqslant K\left(1+\|\varphi\|^{2}\right)
$$

for all $\varphi \in C_{a}\left([-\tau, 0] ; R^{n}\right)$.

Clearly, if we restrict $f$ and $g$ on $C\left([-\tau, 0] ; R^{n}\right)$, Assumptions 2.1 and 2.2 hold; so the theory developed in the previous section holds. But our aim here is to design a simpler 
numerical scheme. To distinguish our new scheme from the one introduced in Section 2, we use the notation $\bar{z}$ and $z$ instead of $\bar{y}$ and $y$, respectively.

The new scheme is formed as follows. Define the discrete approximate solution $\bar{z}(k \Delta)$, $k \geqslant-N$ by

$$
\begin{cases}\bar{z}(k \Delta)=\xi(k \Delta), & -N \leqslant k \leqslant 0, \\ \bar{z}((k+1) \Delta)=\bar{z}(k \Delta)+f\left(\bar{z}_{k \Delta}\right) \Delta+g\left(\bar{z}_{k \Delta}\right) \Delta B_{k}, \quad k \geqslant 0,\end{cases}
$$

where $\bar{z}_{k \Delta}=\left\{\bar{z}_{k \Delta}(\theta):-\tau \leqslant \theta \leqslant 0\right\}$ is a $C_{a}\left([-\tau, 0] ; R^{n}\right)$-valued random variable defined by

$$
\left\{\begin{array}{l}
\bar{z}_{k \Delta}(\theta)=\bar{z}((k-i) \Delta), \quad \text { for }-i \Delta \leqslant \theta<(-i+1) \Delta, i=N,(N-1), \ldots, 1, \\
\bar{z}_{k \Delta}(0)=\bar{z}(k \Delta) .
\end{array}\right.
$$

Clearly, $\bar{z}_{k \Delta}$ is determined only by $\bar{z}((k-N) \Delta), \bar{z}((k-N+1) \Delta), \ldots, \bar{z}(k \Delta)$. Introduce the $C_{a}\left([-\tau, 0] ; R^{n}\right)$-value step process

$$
\bar{z}_{t}=\sum_{k=0}^{\infty} \bar{z}_{k \Delta} 1_{[k \Delta,(k+1) \Delta)}(t), \quad t \geqslant 0,
$$

and define the continuous Euler-Maruyama approximate solution by

$$
z(t)= \begin{cases}\xi(t), & -\tau \leqslant t \leqslant 0 \\ \xi(0)+\int_{0}^{t} f\left(\bar{z}_{s}\right) d s+\int_{0}^{t} g\left(\bar{z}_{s}\right) d B(s), & t \geqslant 0\end{cases}
$$

For any $t \geqslant 0$ and any integer $k \geqslant 0$ that satisfies $k \Delta \leqslant t$, we have

$$
\begin{aligned}
z(t) & =\xi(0)+\int_{0}^{k \Delta} f\left(\bar{z}_{s}\right) d s+\int_{0}^{k \Delta} g\left(\bar{z}_{s}\right) d B(s)+\int_{k \Delta}^{t} f\left(\bar{z}_{s}\right) d s+\int_{k \Delta}^{t} g\left(\bar{z}_{s}\right) d B(s) \\
& =\bar{z}(k \Delta)+\int_{k \Delta}^{t} f\left(\bar{z}_{s}\right) d s+\int_{k \Delta}^{t} g\left(\bar{z}_{s}\right) d B(s)
\end{aligned}
$$

In particular, we observe that $z(k \Delta)=\bar{z}(k \Delta)$ for all $k \geqslant-N$. In the same way as that in which Lemma 3.2 was proved, we can show that the following lemma holds.

Lemma 7.3. Under Assumptions 7.2 and 2.3,

$$
E\left(\sup _{-\tau \leqslant t \leqslant T}|z(t)|^{p}\right) \leqslant H, \quad \forall T>0,
$$

where $H$ is a positive number dependent only on $\xi, K, p$ and $T$, but independent of $\Delta$.

Let us establish another key lemma.

Lemma 7.4. Let Assumptions 7.1, 7.2 and 2.3 hold, and let $T>0$. Then there is a nondecreasing function $\beta:(0, \tau] \rightarrow R_{+}$, which has the property that $\beta(u)=0$ as $u \rightarrow 0$, such that

$$
E\left|z(s+\theta)-\bar{z}_{s}(\theta)\right|^{2} \leqslant \beta(\Delta), \quad s \in[0, T], \theta \in[-\tau, 0] .
$$

Proof. Fix any $s \in[0, T]$ and $\theta \in[-\tau, 0]$. Let $k_{s}$ and $k_{\theta}$ be the integers for which $s \in\left[k_{s} \Delta,\left(k_{s}+1\right) \Delta\right)$ and $\theta \in\left[k_{\theta} \Delta,\left(k_{\theta}+1\right) \Delta\right)$. Clearly, $0 \leqslant s-k_{s} \Delta<\Delta$ and 
$0 \leqslant \theta-k_{\theta} \Delta<\Delta$, so we see that

$$
\begin{aligned}
0 & \leqslant s+\theta-\left(k_{s}+k_{\theta}\right) \Delta \\
& <2 \Delta .
\end{aligned}
$$

Moreover, it follows from (7.5) and (7.6) that

$$
\begin{aligned}
\bar{z}_{s}(\theta) & =\bar{z}_{k_{s} \Delta}(\theta) \\
& =\bar{z}\left(\left(k_{s}+k_{\theta}\right) \Delta .\right.
\end{aligned}
$$

Hence

$$
E\left|z(s+\theta)-\bar{z}_{s}(\theta)\right|^{2}=E\left|z(s+\theta)-\bar{z}\left(\left(k_{s}+k_{\theta}\right) \Delta\right)\right|^{2} .
$$

In the same way as was shown in the four steps in the proof of Lemma 3.3, we can show that

$$
E\left|z(s+\theta)-\bar{z}\left(\left(k_{s}+k_{\theta}\right) \Delta\right)\right|^{2} \leqslant 12 K\left(1+H^{2 / p}\right) \Delta+2 \alpha(2 \Delta),
$$

where $H$ is as specified in Lemma 7.3. The assertion follows immediately.

Using these lemmas, we can show the following theorem in the same way as that in which Theorem 2.5 was proved.

THEOREM 7.5. Under Assumptions 7.1, 7.2 and 2.3,

$$
\lim _{\Delta \rightarrow 0} E\left(\sup _{0 \leqslant t \leqslant T}|x(t)-z(t)|^{2}\right)=0, \quad \forall T>0 .
$$

If we replace the local Lipschitz condition by the global Lipschitz condition given in Assumption 7.6, we can also prove Theorem 7.7, which reveals the order of the convergence.

AssumPTION 7.6. There is a right-continuous nondecreasing function $\mu:[-\tau, 0] \rightarrow R_{+}$ such that

$$
|f(\varphi)-f(\psi)|^{2} \vee|g(\varphi)-g(\psi)|^{2} \leqslant \int_{-\tau}^{0}|\varphi(\theta)-\psi(\theta)|^{2} d \mu(\theta)
$$

for all $\varphi, \psi \in C_{a}\left([-\tau, 0] ; R^{n}\right)$.

THEOREM 7.7. Under Assumptions 7.6 and 5.2,

$$
E\left(\sup _{0 \leqslant t \leqslant T}|x(t)-z(t)|^{2}\right)=O(\Delta), \quad \forall T>0 .
$$

Similarly, if the linear growth condition is replaced by the bounded moment condition given in Assumption 7.8, we have the generalised result given in Theorem 7.9.

Assumption 7.8. For some $p>2$, there is a positive constant $\bar{H}$, which may depend on $\xi, p$ and $T$, but is independent of $\Delta$, such that

$$
E\left(\sup _{-\tau \leqslant t \leqslant T}|x(t)|^{p}\right) \vee E\left(\sup _{-\tau \leqslant t \leqslant T}|z(t)|^{p}\right) \leqslant \bar{H},
$$

THEOREM 7.9. Under Assumptions 7.1, 2.3 and 7.8,

$$
\lim _{\Delta \rightarrow 0} E\left(\sup _{0 \leqslant t \leqslant T}|x(t)-z(t)|^{2}\right)=0, \quad \forall T>0 .
$$




\section{Summary}

Our aim in this work has been to discuss the strong mean-square convergence for the Euler-Maruyama numerical solutions for SFDEs. We give a strong convergence theorem under the local Lipschitz condition and the linear growth condition. When the local Lipschitz condition is replaced by the global Lipschitz condition, we reveal that the order of the convergence is halved, which is the same as in the case of SDEs. A more general convergence theorem is presented for the Euler-Maruyama method in the case where the coefficients of SFDEs are locally Lipschitz and moment bounds are available. A simpler numerical scheme is presented in the case when both coefficients of the underlying SFDE are defined for cadlag functions.

Acknowledgements. The author would like to thank the Royal Society (UK) and the London Mathematical Society for their financial support. The author would also like to thank the referees for their useful suggestions.

\section{References}

1. C. T. H. BAKER and E. BuCKWAR, 'Numerical analysis of explicit one-step methods for stochastic delay differential equations', LMS J. Comput. Math. 3 (2000) 315-335. 141,142

2. E. BUCKWAR, 'Introduction to the numerical analysis of stochastic delay differential equations', J. Comput. Appl. Math 125 (2000) 297-307. 141, 142

3. G. Fleury and P. Bernard, 'Convergence of numerical schemes for stochastic differential equations', Monte Carlo Methods Appl. 7 (2001) 35-44. 141

4. I. Gyöngy, 'A note on Euler's approximations', Potential Anal. 8 (1998) 205-216. 141

5. I. GYÖNGY and N. KRYLOV, 'Existence of strong solutions for Itô's stochastic equations via approximations', Probab. Theory Related Fields 105 (1996) 143-158. 141

6. J. K. HALE and V. S. M. Lunel, Introduction to functional differential equations (Springer, Berlin, 1993). 141

7. D. J. Higham, X. MAo and A. M. StUART, 'Strong convergence of numerical methods for nonlinear stochastic differential equations', SIAM J. Numer. Anal. 40 (2002) 10411063. 141, 142

8. Y. Hu, 'Semi-implicit Euler-Maruyama scheme for stiff stochastic equations', Stochastic analysis and related topics V: The Silvri Workshop, Progr. Probab. 38 (ed. H. Koerezlioglu, Birkhauser, Boston, MA, 1996) 183-302. 141, 142

9. Y. Hu, S. E. A. Mohammed and F. YAN, 'Numerical solution of stochastic differential systems with memory', Preprint, Southern Illinois University, 2001. 141, 142

10. P. E. KLOEDEN and E. PlATEN, Numerical solutions of stochastic differential equations (Springer, Berlin, 1992). 141, 142

11. V. KolmanovskiI and A. Myshkis, Applied theory of fundamental differential equations (Kluwer Academic Publishers, Dordrecht, 1992).

12. U. Küchler and E. Platen, 'Strong discrete time approximation of stochastic differential equations with time delay', Math. Comput. Simulation 54 (2000) 189-205. 141,142 
13. X. Mao, Approximate solutions for a class of stochastic evolution equations with variable delays-part II, Numerical Functional Analysis and Optimisation 15 (1994) 65-76. 141,142

14. X. MAo, Exponential stability of stochastic differential equations (Marcel Dekker, New York, 1994). 141, 142

15. X. Maо, Stochastic differential equations and applications (Horwood, England, 1997). $141,142,143$

16. $X$. MAO and S. SABANIS, 'Numerical solutions of stochastic differential delay equations under local Lipschitz condition', J. Comput. Appl. Math. 151 (2003) 215-227. 142

17. G. N. MilsteIn, Numerical integration of stochastic differential equations (Kluwer Academic Publishers Group, Dordrecht, 1995). 141, 142

18. E. Platen, 'An introduction to numerical methods for stochastic differential equations', Acta Numerica 8 (1999) 195-244. 141, 142

Xuerong Mao xuerongestams.strath.ac.uk

http://www.stams.strath.ac.uk/ xuerong

Department of Statistics and Modelling Science

University of Strathclyde

Glasgow G1 1XH 